\title{
Global neurosurgery: a scoping review detailing the current state of international neurosurgical outreach
}

\author{
Anthony T. Fuller, MD, MScGH,1-4 Ariana Barkley, MD, ${ }^{1,5}$ Robin Du, BS, ${ }^{1,6}$ Cyrus Elahi, MScGH, ${ }^{1,3,7}$ \\ Ali R. Tafreshi, BA, ${ }^{1,6}$ Megan Von Isenburg, MSLS, ${ }^{2}$ and Michael M. Haglund, MD, PhD, MACM ${ }^{1-4}$ \\ 1Duke University Division of Global Neurosurgery and Neurology; '2Duke University School of Medicine; ${ }^{3}$ Duke University \\ Global Health Institute; ${ }^{4}$ Department of Neurosurgery, Duke University Medical Center, Durham, North Carolina; ${ }^{5}$ Department \\ of Neurological Surgery, University of Washington, Seattle, Washington; ${ }^{6 K}$ Keck School of Medicine of the University of Southern \\ California, Los Angeles, California; and PPaul L. Foster School of Medicine, El Paso, Texas
}

\begin{abstract}
OBJECTIVE Global neurosurgery is a rapidly emerging field that aims to address the worldwide shortages in neurosurgical care. Many published outreach efforts and initiatives exist to address the global disparity in neurosurgical care; however, there is no centralized report detailing these efforts. This scoping review aims to characterize the field of global neurosurgery by identifying partnerships between high-income countries (HICs) and low- and/or middle-income countries (LMICs) that seek to increase neurosurgical capacity.
\end{abstract}

METHODS A scoping review was conducted using the Arksey and O'Malley framework. A search was conducted in five electronic databases and the gray literature, defined as literature not published through traditional commercial or academic means, to identify studies describing global neurosurgery partnerships. Study selection and data extraction were performed by four independent reviewers, and any disagreements were settled by the team and ultimately the team lead.

RESULTS The original database search produced 2221 articles, which was reduced to 183 final articles after applying inclusion and exclusion criteria. These final articles, along with 9 additional gray literature references, captured 169 unique global neurosurgery collaborations between HICs and LMICs. Of this total, $103(61 \%)$ collaborations involved surgical intervention, while local training of medical personnel, research, and education were done in $48 \%, 38 \%$, and $30 \%$ of efforts, respectively. Many of the collaborations (100 [59\%]) are ongoing, and $93(55 \%)$ of them resulted in an increase in capacity within the LMIC involved. The largest proportion of efforts began between 2005-2009 (28\%) and 2010-2014 (17\%). The most frequently involved HICs were the United States, Canada, and France, whereas the most frequently involved LMICs were Uganda, Tanzania, and Kenya.

CONCLUSIONS This review provides a detailed overview of current global neurosurgery efforts, elucidates gaps in the existing literature, and identifies the LMICs that may benefit from further efforts to improve accessibility to essential neurosurgical care worldwide.

https://thejns.org/doi/abs/10.3171/2020.2.JNS192517

KEYWORDS global health; global neurosurgery; capacity development

$\mathrm{S}$ AFE, accessible, and affordable surgical services have become an inherent component of the global discourse to address the burden of disease worldwide and fully realize the lofty goal of universal healthcare. In 2000, the United Nations put forth their Millennium Development Goals with the primary healthcare focus on infectious disease and maternal health. When the Millennium Development Goals were set to expire in 2015, the United Nations implemented the global Sustainable Devel- opment Goals for 2030. ${ }^{1}$ This new outline afforded a shift in the global health paradigm to incorporate the "neglected stepchild of global health": surgical services. ${ }^{2}$

According to The Lancet Commission on Global Surgery, surgically treatable conditions are responsible for over $30 \%$ of the global burden of disease. An estimated 5 billion people lack access to surgical care when needed, with the greatest disparity in low- and/or middle-income countries (LMICs), as defined by the World Bank. ${ }^{3}$ Al-

ABBREVIATIONS HIC = high-income country; LMICs = low- and/or middle-income countries .

SUBMITTED September 13, 2019. ACCEPTED February 28, 2020.

INCLUDE WHEN CITING Published online May 8, 2020; DOI: 10.3171/2020.2.JNS192517. 
though neurosurgery was once colloquially considered a "luxury subspecialty," the rising burden of neurosurgical pathology means that the specialty is now included within essential surgical services. ${ }^{3,4}$ A recent summary of the global neurosurgical burden revealed that 13.8 million patients suffer from neurological pathology requiring neurosurgical intervention. ${ }^{5}$ Traumatic brain injury, strokerelated conditions, tumors, hydrocephalus, and epilepsy constitute the majority of the 5 million essential neurosurgical cases annually. To meet this demand, researchers estimate that over 20,000 additional neurosurgeons are needed. ${ }^{5}$

Several strategies have emerged to address the global disparity in neurosurgical care. Many published initiatives exist in the context of partnerships between high-income country (HIC) institutions or organizations and those in the LMIC setting that support projects such as surgical camps, training and education, research endeavors, establishment of residency programs or fellowships, and international conferences. The concept of "twinning" an HIC to an LMIC institution has therefore emerged as a central theme around initiatives aimed toward improving access to this essential surgical service. ${ }^{6}$ In fact, the development of an LMIC's capacity to safely provide neurosurgical interventions can lead to a concomitant increase in its capacity to perform many other essential surgical services. ${ }^{6}$ Crossinstitutional organizations such as the Foundation for International Education in Neurological Surgery (FIENS), World Federation of Neurosurgical Societies (WFNS), and College of Surgeons of East, Central, and Southern Africa (COSECSA) are some examples of attempts to organize international efforts. Despite the plethora of individual organizations, twinning partnerships, and capacitybuilding global neurosurgery initiatives, there is no central repository or report detailing these efforts nor a review that evaluates the geographic distribution of initiatives.

After considering a variety of systematic review approaches for reporting current global neurosurgery efforts, we chose to conduct a scoping review, drawing on Arksey and O'Malley's seminal methodological framework ${ }^{7}$ and subsequent studies that have posed recommendations to expand on this framework. ${ }^{8,9}$ Compared to the more standard systematic review, the scoping review is particularly useful for addressing broader topics with heterogeneous study designs or publication formats and can be used to identify research gaps in the existing literature. Global neurosurgery as a field is relatively new, and many partnerships have not published results in peer-reviewed journals; these factors fit well with the utility of scoping reviews.

The aims of this review were to identify efforts in and approaches to global neurosurgery in the context of twinning partnerships; to determine which HICs and LMICs are involved, allowing acknowledgment of LMICs that may not be included in current initiatives; and, finally, to determine gaps in the literature as well as the feasibility of future systematic reviews examining impact and sustainability. The results garnered from this review not only serve as an initial step in reporting global neurosurgery initiatives, and therefore as a resource for those seeking to become involved, but also shed light on LMICs that stand to benefit from increased efforts to improve neurosurgical capacity.

\section{Methods}

The scoping review framework is a five-stage approach, with our applications for each stage and relevant recommendations discussed below. We used an iterative team approach "requiring researchers to engage with each stage in a reflexive way and, where necessary, repeat steps to ensure that the literature is covered in a comprehensive way." 7 A review protocol is not publicly available.

\section{Stage 1: Identifying the Research Question}

Our scoping review was guided by the following questions: What global neurosurgery work has been published? What are the ways that individuals, institutions, and other entities engage in global neurosurgery initiatives? For the purposes of this study, we defined a "global neurosurgery initiative" as any collaboration between an HIC and an LMIC to deliver, develop, or study neurosurgical care in an LMIC, using the World Bank definition for the economic classifications of countries. Any and all procedures of the central nervous system, peripheral nervous system, and spine were included within the category of neurosurgery.

\section{Stage 2: Identifying Relevant Studies}

Our search was conducted in five electronic databases: PubMed, Embase, Scopus, Web of Science, and regional indices in the Global Health Library (Western Pacific Region Index Medicus [WPRIM], Latin America and the Caribbean Literature on Health Sciences [LILACS], Index Medicus for the Eastern Mediterranean Region [IMEMR], and Index Medicus for the South-East Asia Region [IMSEAR]). Search strategies were developed and run by our librarian team member (M.V.I.) to include keywords and database-specific subject headings for neurosurgical procedures, partnerships, and LMICs. Results were imported, de-duplicated, and organized using the citation manager EndNote. In addition, we conducted a gray literature search, defined as a search for literature published through means other than traditional commercial or academic channels, using three search engines (Google, Bing, and Yahoo!) and the following search phrases: "neurosurgery mission trips," "neurosurgery missions," "global neurosurgery." The gray literature search results were cross-referenced with results from the initial database search to eliminate duplicates.

\section{Stage 3: Study Selection}

We used a two-stage screening process to select studies to include from the initial database search results. In pairs of two, team members first reviewed the studies for relevance and eligibility for inclusion, with any disagreements discussed with the team and settled by the team lead (A.T.F.). We subsequently conducted the second stage of screening using the below inclusion and exclusion criteria. Studies that fulfilled at least one inclusion criterion and did not fulfill any exclusion criteria were included in our review.

Inclusion criteria consisted of the following: effort described is undertaken by an HIC and is being implemented physically or virtually in an LMIC, defined as low, lower-middle, and upper-middle income economies 
TABLE 1. Variables included in the analysis

\begin{tabular}{|c|c|}
\hline Variable & Definition \\
\hline Publications reviewed & Final no. of articles included in data extraction \\
\hline Journal totals & Top 5 journals publishing articles included in this study \\
\hline Collaboration start yr & Yr the HIC-LMIC collaboration began \\
\hline Publication yr & Yr the article published \\
\hline Known collaborations & Collaboration in which $1 \mathrm{HIC}$ institution could be mapped to $1 \mathrm{LMIC}$ institution \\
\hline HICs involved & No. of unique HICs involved in global neurosurgery work \\
\hline LMICs involved & No. of unique LMICs involved in global neurosurgery work \\
\hline HIC institutions involved (known) & $\begin{array}{l}\text { No. of unique HIC institutions involved in global neurosurgery work ("known" indicates the HIC institution is linked } \\
\text { to } 1 \text { LMIC institution) }\end{array}$ \\
\hline LMIC institutions involved (known) & $\begin{array}{l}\text { No. of unique LMIC institutions involved in global neurosurgery work ("known" indicates the LMIC institution is } \\
\text { linked to } 1 \mathrm{HIC} \text { institution) }\end{array}$ \\
\hline Intervention type & $\begin{array}{l}\text { Global neurosurgery efforts categorized as } 1 \text { or more of the following: surgery, training, research, education, } \\
\text { resource building, or telemedicine }\end{array}$ \\
\hline Project status & Global neurosurgery efforts categorized as ongoing, stopped, or could not be determined \\
\hline Capacity development & $\begin{array}{l}\text { Capacity at LMIC institution defined as increased, unchanged, or unknown based on the report; "capacity build- } \\
\text { ing" defined as an intervention that produced significant increase in ability of LMIC facilities to deliver care that } \\
\text { lasts beyond the context of original intervention }\end{array}$ \\
\hline
\end{tabular}

by the World Bank Country and Lending Groups; effort described includes surgical operation, nonsurgical consultation, and/or capacity building performed by at least one neurosurgeon from an HIC; and effort described consists of research conducted by an HIC regarding the state of neurosurgery in an LMIC.

Exclusion criteria consisted of the following: effort described consists of neurosurgical work by an HIC in a military setting conducted exclusively for HIC military personnel; effort described consists of research regarding the state, history, or growth of neurosurgery in an LMIC but does not describe any specific efforts; and an editorial or opinion piece that describes what should or could be done to improve neurosurgery in an LMIC but does not describe any specific efforts.

\section{Stage 4: Charting the Data}

Four team members (C.E., R.D., A.R.T., A.B.) participated in the review and extraction of data. The reviewers extracted the following data from the included studies and entered them into a spreadsheet: article identifiers (title, authors, publication year, journal), study components (study population, types of surgery/neurosurgery performed, sample size, study/research design, type of intervention, start and end date, HIC and LMIC names and institutions), and study results (outcomes measured, number of patients treated, number of residents trained, study ongoing vs stopped, whether capacity was built). Following the recommendation of Daudt and colleagues to conduct a trial charting exercise, each of the four reviewers extracted data from the same five studies. ${ }^{8}$ This step allowed us to determine whether the approach to data extraction was consistent with the research question and purpose. Following this, we divided the four reviewers into two teams of two to ensure that each article had a minimum of two reviewers for data extraction. We discussed disagreements as a team, with unresolved disagreements settled by the team lead (A.T.F.).

\section{Stage 5: Collating, Summarizing, and Reporting the Results}

We used a predominantly quantitative approach to collate, summarize, and map the literature. Data collection included focused and broad data elements to answer our research questions and support future investigations of global neurosurgery efforts. After data collection, we identified variables that had low numbers of missing values, assisted in answering our research question, and could promote hypothesis generation for future studies. We provide the final variables and variable definitions from the analysis in Table 1. We omitted variables with high numbers of missing values or that did not lend themselves to uniform data collection. We completed all analysis and figure generation using the computing environment R (R 3.5.2, R Foundation for Statistical Computing, http://www.R-project.org/).

\section{Stage 6: Consultation}

Arksey and O'Malley's methodological framework suggests an optional sixth stage of consultation with key stakeholders, ${ }^{7}$ whereas Levac and colleagues assert that this step is an essential component that should be required. ${ }^{9} \mathrm{We}$ identified key stakeholders involved in global neurosurgery and sent our initial data chart for review. We asked for their general feedback and to inform us of any HIC-LMIC global neurosurgery partnerships that we may have missed during our data extraction.

\section{Results}

\section{Article Inclusion and Source Identification}

Our initial database inquiry (Fig. 1) yielded a total of 2221 peer-reviewed articles. During the title/abstract re- 


\section{Database Search}

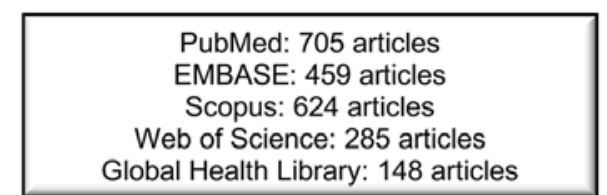

Search terms associated with neurosurgery, names of LMICs, and an intervention

Web of Science: 285 articles

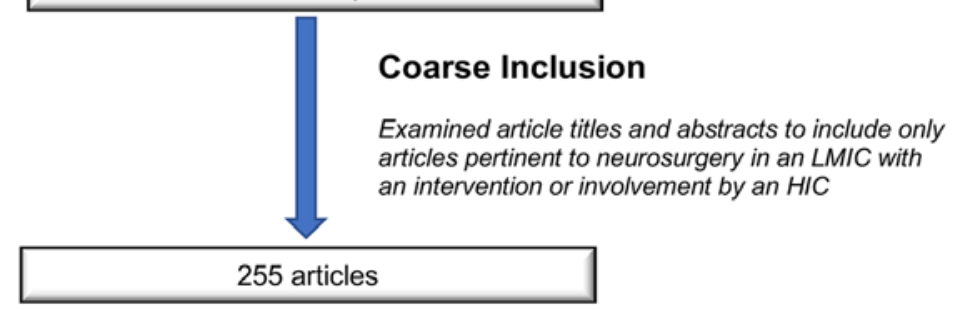

\section{Granular Inclusion and Exclusion}

Examined article titles, abstracts, and manuscripts to include articles that fit at least one of the following inclusion criteria:

1. The article describes an effort undertaken by an HIC that is being implemented physically or virtually in an LMIC

2. Surgical operation, non-surgical consultation, and/or capacity building done by at least one neurosurgeon from an HIC is a part of the effort described in the article

3. The article describes research regarding the state of neurosurgery in an LMIC conducted by an HIC

and exclude articles that fit any of the following exclusion criteria:

1. The article describes HIC military neurosurgical work done in an LMIC exclusively for HIC personnel

2. The article describes research regarding the state of neurosurgery in an LMIC conducted without involvement by an HIC

3. The article is an editorial that does not describe actionable effort

4. The article describes the history and growth of neurosurgery in an LMIC without involvement from an HIC

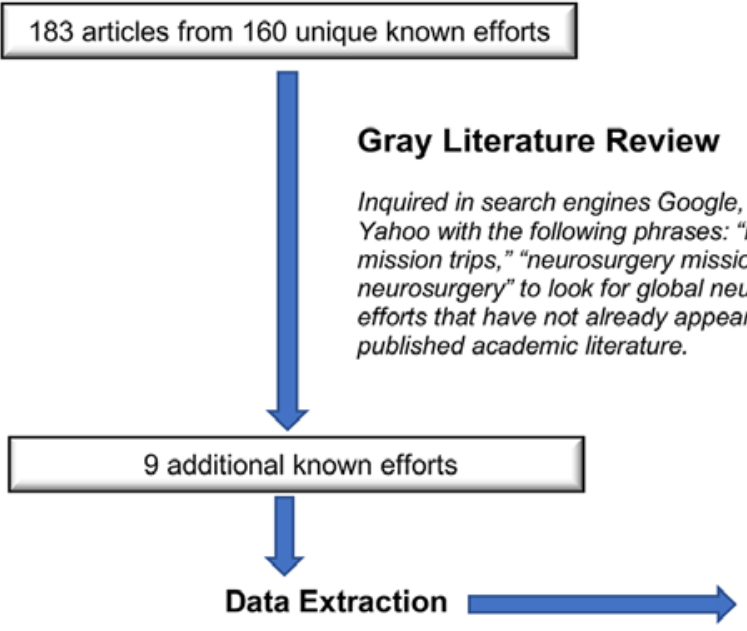

\section{Expert Consultation}

We asked a panel of experts in the field of global neurosurgery at our institutions to grossly verify the accuracy of the efforts and to add to our list any efforts that were unaccounted for.

FIG. 1. Scoping review stages. Figure is available in color online only.

view inclusion step, we removed articles whose titles and abstracts were not related to global neurosurgery, leaving a total of 255 articles. From that total, we culled 183 articles after applying the appropriate inclusion and exclusion criteria. Next, we conducted a gray literature search using the three most popular internet search engines (Google, Bing, and Yahoo!), which yielded a total of 9 relevant references. Upon examination of the relevant articles and gray literature references, our team identified a total of 169 unique, known global neurosurgical efforts. After collecting the relevant data, we sent them to experts in global neurosurgery before finalizing our results. 
TABLE 2. General information on articles extracted during the scoping review

\begin{tabular}{|c|c|}
\hline Category & No. $(\%)$ \\
\hline Total no. publications reviewed & 183 \\
\hline \multicolumn{2}{|l|}{ Journal } \\
\hline World Neurosurgery & $68(37)$ \\
\hline Journal of Neurosurgery: Pediatrics & $17(9)$ \\
\hline Journal of Neurosurgery & $12(7)$ \\
\hline Child's Nervous System & $6(3)$ \\
\hline Neurosurgery & $6(3)$ \\
\hline Other* & $74(40)$ \\
\hline \multicolumn{2}{|l|}{ Collaboration start yr } \\
\hline Before 1990 & $14(8)$ \\
\hline 1990-1994 & $2(1)$ \\
\hline 1995-1999 & $6(3)$ \\
\hline 2000-2004 & $27(15)$ \\
\hline $2005-2009$ & $51(28)$ \\
\hline $2010-2014$ & $32(17)$ \\
\hline $2015-2019$ & $11(6)$ \\
\hline Not available & $40(22)$ \\
\hline \multicolumn{2}{|l|}{ Yr published } \\
\hline Before 2000 & $5(3)$ \\
\hline $2000-2004$ & $6(3)$ \\
\hline 2005-2009 & $19(10)$ \\
\hline 2010-2014 & $80(44)$ \\
\hline 2015-2019 & $73(40)$ \\
\hline
\end{tabular}

* None of the journals in the "other" category had more than 4 articles published.

\section{Publication Information}

The majority of articles (84\%) were published in 2010 or later (Table 2). The journals that most commonly published articles pertaining to global neurosurgery were World Neurosurgery, followed by the Journal of Neurosurgery: Pediatrics and the Journal of Neurosurgery. The articles in our review included a wide range of study and publication types including case reports, case series, retrospective cohort studies, cross-sectional studies, case-control studies, review articles, technical papers, miscellaneous chart reviews, outcome studies, field reports, editorials, comparative analyses, predictive research, and review articles.

\section{Unique Neurosurgical Efforts}

The largest proportion of collaborative efforts began between 2005-2009 (28\%) and 2010-2014 (17\%). The efforts featured surgery (a partnership in which HIC providers work in an LMIC for a short period of time providing neurosurgical services), local resource building, LMIC personnel education, LMIC personnel surgical training, research, and telemedicine services (Table 3). Many efforts involved a combination of these various interventions. The most common intervention types were surgery, local training, and research.

\section{Capacity Development and Continuity}

In addition to intervention type, we examined whether
TABLE 3. Global neurosurgery collaborations

\begin{tabular}{lc}
\hline \multicolumn{1}{c}{ Category } & No. \\
\hline Collaborations & \\
\hline Known collaborations* & 169 \\
\hline HICs & 23 \\
\hline LMICs & 45 \\
\hline Total institutions in HICs (known) & $171(117)$ \\
\hline Total institutions in LMICs (known) & $110(85)$ \\
\hline Intervention type & \\
\hline Surgery & 103 \\
\hline Training & 81 \\
\hline Research & 64 \\
\hline Education & 50 \\
\hline Resource building & 18 \\
\hline Telemedicine & 3 \\
\hline Project status & $100(59 \%)$ \\
\hline Ongoing & $40(24 \%)$ \\
\hline Discontinued & $29(17 \%)$ \\
\hline Could not be determined & \\
\hline Capacity development at LMIC institution & $53(55 \%)$ \\
\hline Increased & $58(34 \%)$ \\
\hline Unchanged & $18(11 \%)$ \\
\hline Unknown & \\
\hline$*$ Known colarions had an identfab HI and & \\
\hline
\end{tabular}

* Known collaborations had an identifiable HIC and LMIC institution.

an effort contributed to capacity development in the LMIC, which was defined as the ability to autonomously continue to provide neurosurgical services. This included but was not limited to local education, local resource building, or other enhancements to the medical infrastructure in the LMIC not otherwise specified. In this regard, we found that $93(55 \%)$ efforts suggested capacity development, while $58(34 \%)$ did not. For the remaining 18 (11\%), we did not have sufficient information to determine whether the effort resulted in capacity development in the LMIC (Table 3).

At the time of this study, 100 (59\%) efforts were ongoing, while 40 (24\%) had been indefinitely terminated; we did not have sufficient information to accurately determine whether the remaining $29(17 \%)$ efforts were ongoing at the time of our review (Table 3 ).

Our ability to determine the total number of persons (patients, healthcare personnel, and/or community members) affected by the efforts was inherently limited given the lack of detailed information on the number of participants in each effort (e.g., reports that make mention of service trips but do not give the total number of patients and healthcare providers involved). In applicable studies, sample sizes ranged from 1 to 6735 .

\section{HIC-LMIC Collaborations}

HICs participating in collaborations were concentrated in Europe and the Americas, while the most frequently involved LMICs were primarily located in Africa, South America, and Southeast Asia (Fig. 2). The HIC with the greatest number of collaborations was the United States of 
Fuller et al.
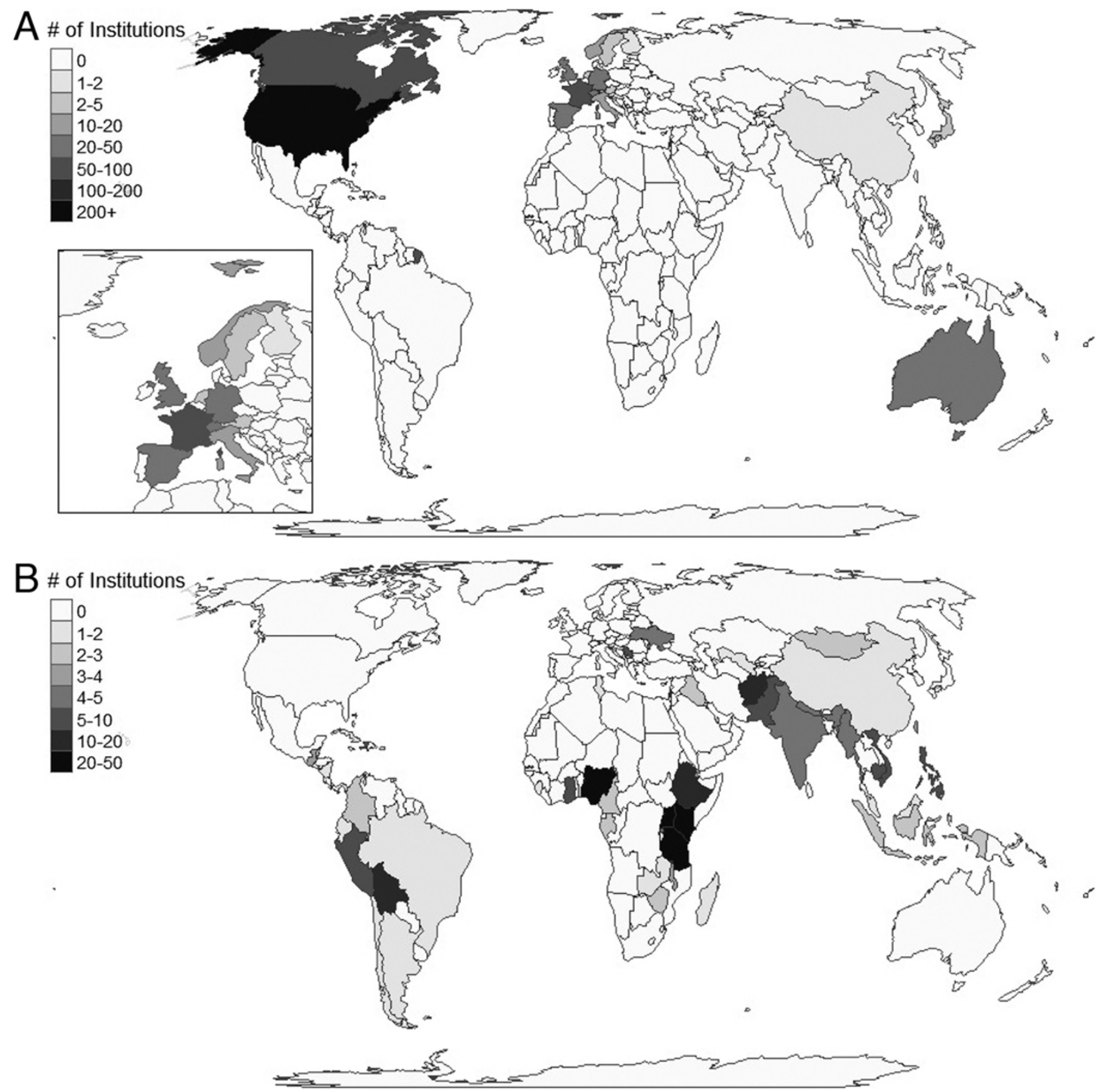

FIG. 2. A choropleth, a thematic map indicating the concentration of global neurosurgery efforts for institutions shaded in proportion to the number of institutions involved in collaborations in HICs (A) and LMICs (B). The R software package tmap v3.0 was used to produce this map (R Foundation for Statistical Computing).

America, followed by Canada, France, United Kingdom, and Australia (Fig. 3). The top 5 LMICs with the greatest number of collaborations were all in Africa-specifically, Uganda, followed by Tanzania, Kenya, Nigeria, and Ethiopia.

\section{Discussion}

Our scoping review provides the first characterization of initiatives in the growing field of global neurosurgery and the HICs as well as the LMICs involved in these international partnerships. Intervention types ranged from surgical camps to training and research partnerships to donations of equipment and resources, as well as telemedicine services. A majority of the efforts included in our review were ongoing (59\%), which speaks to the increased emphasis on continuous efforts as opposed to one-time interventions (Table 3 ).

The long-term impact of a particular effort is a major 


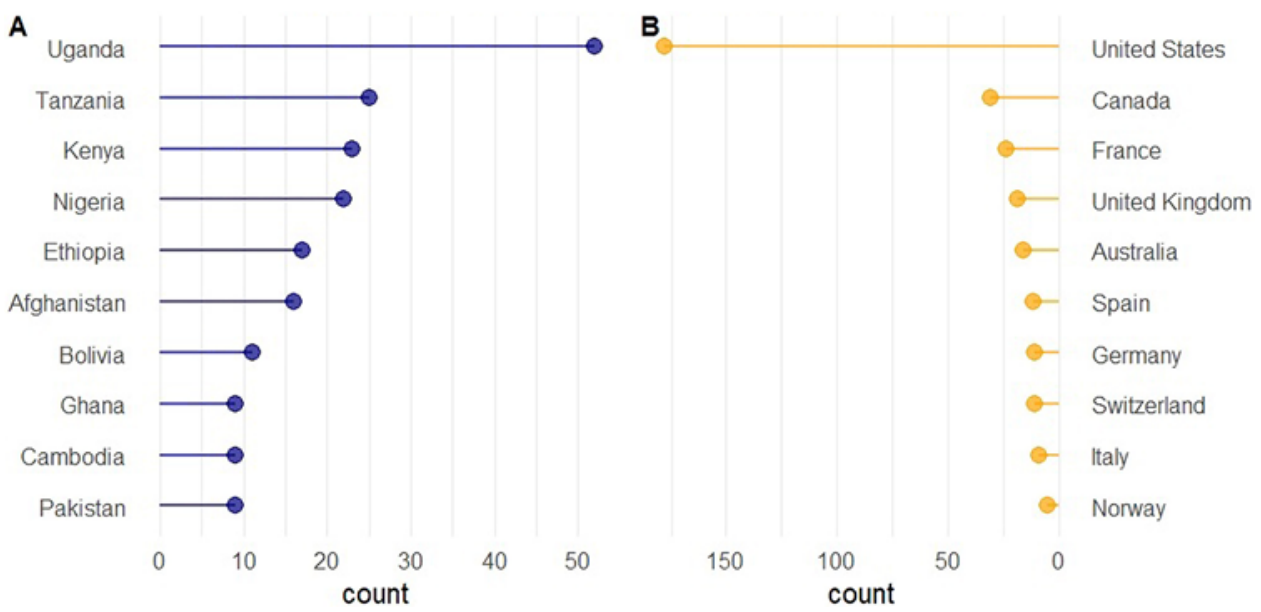

FIG. 3. A lollipop chart identifying the number of institutions/organizations within the top 10 LMICs $(\mathbf{A})$ and HICs (B) involved in global neurosurgery efforts. Figure is available in color online only.

consideration when discussing global outreach. We characterized the efforts identified in our review by intervention type, for example, surgical camps conducted by HIC-LMIC partners, local personnel training, educational sessions, or provision of medical resources and supplies. However, intervention type did not necessarily reflect neurosurgical "capacity development," defined as the ability to autonomously continue to provide neurosurgical services. While interventions that involve training, education, or resource building lend themselves to long-lasting impact, these efforts do not always build long-term capacity in the LMIC. One example is a telemedicine consultation program that trained the local community on the use of teleconferencing technologies, which was subsequently removed upon termination of the effort $;^{10}$ such training, while integral to the operation of the effort, is unlikely to lead to building capacity.

Conversely, the fact that an effort is discontinued does not preclude it from being a capacity-building initiative. Forty (24\%) of the efforts in our review were classified as discontinued (Table 3). Some of them included efforts tied to a neurosurgeon from an HIC who had been living in an LMIC for a period of time but at some point returned to their home country, with subsequent termination of their interventions but successful transference of skills to local healthcare providers. Other currently discontinued efforts included research surveys or projects that had a concrete end date, military efforts that concluded at the end of the war period, or training programs to enhance the skill level of LMIC doctors by exposing them to international patient populations. These observations suggest that successful capacity development, defined by the United Nations Development Programme (UNDP) as "bring[ing] about transformation that is sustained from within," "relies less on intervention type per our definition and more on initiative and commitment from the native country in question supported by resources to accomplish their capacitydevelopment initiatives. This is supported by the UNDP's published steps in the capacity-development process. ${ }^{11}$

The long-term impact of these and other efforts is difficult to address in this study, namely because we are lim- ited by the fact that this variable was not included in the original articles and because related follow-up studies to assess long-term impact are lacking; however, our review demonstrates that the surgeries or research conducted as a result of these efforts does lead to care given to patients in LMICs that would otherwise be unavailable. Such efforts have a clear and significant impact on the local delivery of neurosurgical care and suggest that an in-depth examination of the longitudinal effects of global neurosurgery interventions is warranted, with emphasis on determining the effects on local capacity development.

We have demonstrated that initiatives and partnerships between HICs and LMICs aimed toward improving accessibility to neurosurgical services have increased as of 2005-2009 (Table 2). This correlates with the reported increase in isolated articles and editorials calling for the global health community to incorporate surgical initiatives in global health priorities. ${ }^{4}$ Publications highlighting these efforts also increased between 2010 and 2014, with over $80 \%$ of the total reviewed articles published since 2010. Articles were predominantly published in World Neurosurgery and the Journal of Neurosurgery: Pediatrics. Pediatric specialty journals were relatively overrepresented, composing two of the top five journals. This propensity may be attributable to a variety of factors: an increased willingness of the pediatric neurosurgical community to participate in and/or support global neurosurgery efforts, a higher burden of pediatric disease in LMICs amenable to interventions that can be performed in a limited resource setting, and a larger possible patient population allowing scientific study leading to a higher rate of publication in said settings. In general, the rise in the number of initiatives and publications demonstrates a growing interest in global neurosurgery, which parallels its increased prominence in the global health dialogue.

We discovered that despite the increase in the number of global neurosurgery initiatives, there is a noticeable concentration between a handful of HICs and LMICs. As defined by the World Bank, there are currently 81 countries classified as "high income" and 71 countries classified as either "low income" or "low/middle income." 
Of the known collaborations identified in our review, 23 (28.4\%) of the 81 HICs collaborated with $45(63.4 \%)$ of the 71 LMICs (Table 3). The top 3 HICs involved in these partnerships were the United States, Canada, and France (Figs. 2A and 3), while the top 3 LMICs were Uganda, Tanzania, and Kenya, all located in East Africa (Figs. 2B and 3). In fact, 6 of the top 10 LMICs were in Africa; the other 4 countries were Afghanistan, which had published collaborations only in a military context, Bolivia, Pakistan, and Cambodia. This finding may be attributable to the fact that Africa has the greatest concentration of lowincome countries, followed by Southeast Asia and South America. Furthermore, Africa has access to less than $1 \%$ of the practicing neurosurgeons worldwide despite accounting for $15 \%$ of the global volume of neurosurgical disease. ${ }^{12}$ The concentration of efforts in LMICs revealed in this review is useful for informing geographic suggestions for future initiatives. If the main goal is to provide equitable access to these resources, there is a strong argument for starting new initiatives in interested LMICs composing the 28 remaining countries without this type of international partnership.

The assumption that a high concentration of international collaborations in a single LMIC would lead to the improvement of neurosurgical capacity is not necessarily accurate and is something that would be better explored within a systematic review or a specific research project. The collaboration of multiple HIC institutions or countries with a single LMIC institution may lead to a diffusion of resources or a lack of focused effort. There may also be a fractionation of resources toward several different and potentially discordant goals. Conversely, there may be an unknown duplication of efforts between multiple organizations or institutions. However, one can surmise that LMICs lacking the same degree of collaboration may not have access to the same resources necessary for quickly building a sustainable infrastructure capable of providing neurosurgical services. This reflects the value of not only supporting collaborations with this ultimate goal but also organizing initiatives to optimize capacity development.

The ultimate goal of these international collaborations is to decrease the global disparity in neurosurgical care by increasing the capacity to provide these services in LMIC settings. There is a dearth of literature, however, evaluating the effectiveness of each of these initiatives, which is further challenged by the lack of an agreed-upon standard of measurement of neurosurgical capacity. Measurements such as the number of practicing neurosurgeons, the number of neurosurgical procedures performed, and adherence to surgical assessment tools such as the WHO personnel, infrastructure, procedures, equipment, and supplies (PIPES) checklist, in addition to the availability of specific surgical tools, have all been used as proxies for measuring neurosurgical capacity in LMICs. ${ }^{13}$ Equally important are perioperative outcomes such as functional status and follow-up care, which are intimately influenced by the presence of ancillary supportive services as well as the availability of assistive devices. Further analysis of the outcomes of these initiatives on a range of these measures is required to determine whether well-meaning international collaborations are achieving the end goal of increas- ing the capacity of LMICs to provide safe and effective neurosurgical care.

One potential solution to establishing a globally agreedupon means of measuring neurosurgical capacity would be to build on the six global surgical indicators already described by The Lancet Commission. These include geographic accessibility of surgical facilities, density of specialist surgical providers, number of surgical procedures provided per 100,000 population, perioperative mortality rates, the risk of impoverishing expenditure when surgery is required, and the risk of catastrophic expenditure when surgery is required..$^{14,15}$ However, these indicators are more long-term measures that smaller or beginning efforts may not influence for a substantial period of time. Therefore, adjusting these measurements to reflect shorter-term effects of global neurosurgery initiatives and determining the optimal timing of analysis to existing efforts would be helpful to determine which types of interventions are considered most effective. This may help to streamline strategies to efficiently decrease global disparities in neurosurgical access.

As we continue to support efforts aimed toward decreasing global disparity in neurosurgical care, we must consider disease prevalence in addition to the social and economic environment in which interventions are introduced. Balancing subspecialty and general healthcare infrastructure development will be unique to the needs of each nation. These can be taken into account through evaluations such as needs assessments and epidemiological assessments of disease in addition to determinations of the financial cost of introducing the infrastructure necessary to provide neurosurgical care.

\section{Study Limitations}

Scoping reviews have inherent limitations given their design. Most pertinent to this review is the fact they do not include an assessment of the quality of included studies, nor do they address the relative weight of evidence. ${ }^{7-9}$ Thus, the nature of the included studies precludes a formal thematic analysis given differences in the detail, quality, and focus of included papers and projects. These limitations may be overcome with future systematic reviews that evaluate these factors and highlight projects with longterm follow-up to identify characteristics of interventions that lend themselves to success as it will be defined in this emerging academic field.

Our review contains an inherent selection bias given that articles were screened primarily in English and limited to five electronic databases (Embase, Global Health Library, PubMed, Scopus, and Web of Science). The gray literature review was also limited to three English internet search engines (Google, Bing, and Yahoo!). Since our review aimed to characterize the types and number of collaborations, it is important to note that, given these limitations, this review may not fully reflect the total body of literature published in global neurosurgery.

A lack of detail in some included articles, abstracts, and gray literature results did not allow characterization or definition of exact HIC-LMIC partnerships, leaving $21 \%$ unknown. Some of the gray literature review results consisted of merely a sentence or two in the biography of 
a neurosurgeon stating his or her involvement in global neurosurgery but no additional details, whereas some of the included abstracts contained minimal detail about the partnership and initiative. In addition, we recognize that there likely exist partnerships and projects that have not been described or reported in the gray or published literature, which biases results to include academic groups and larger, more well-known philanthropic institutions. Despite these limitations, the scoping review is a rigorous and extensive means of examining the extent, range, and nature of research activity, making it a fitting approach to summarize and map current global neurosurgery initiatives.

\section{Conclusions}

Our review provides a detailed overview of current global neurosurgery efforts, elucidates gaps in the existing literature, and identifies LMICs that may benefit from further efforts to improve the accessibility to essential neurosurgical care worldwide. This review provides the first detailed account of the ways in which people, organizations, and academic institutions are engaging in global neurosurgery. Surgical camps, training, and research partnerships stand out as the primary methodologies used, with an increasing emphasis on long-term impact and sustainability. Despite this emphasis, a unifying ideology for assessing global neurosurgery partnerships is lacking, precluding a more in-depth comparison to determine best practices. Global neurosurgery is a growing field that has rapidly expanded in the last decade with complex partnerships between HICs and LMICs; this complexity requires the field to understand where partnerships already exist, examine its strategies, develop a common language, and work collaboratively to improve access to and the quality of neurosurgical services globally.

\section{References}

1. Campbell DA. An update on the United Nations Millennium Development Goals. J Obstet Gynecol Neonatal Nurs. 2017;46(3):e48-e55.

2. Farmer PE, Kim JY. Surgery and global health: a view from beyond the OR. World J Surg. 2008;32(4):533-536.

3. Meara JG, Leather AJ, Hagander L, et al. Global Surgery 2030: evidence and solutions for achieving health, welfare, and economic development. Lancet. 2015;386(9993):569624.

4. Park KB, Johnson WD, Dempsey RJ. Global neurosurgery: the unmet need. World Neurosurg. 2016;88:32-35.

5. Dewan MC, Rattani A, Fieggen G, et al. Global neurosurgery: the current capacity and deficit in the provision of essential neurosurgical care. J Neurosurg. 2019;130(4):10551064.
6. Haglund MM, Kiryabwire J, Parker S, et al. Surgical capacity building in Uganda through twinning, technology, and training camps. World J Surg. 2011;35(6):1175-1182.

7. Arksey H, O’Malley L: Scoping studies: towards a methodological framework. Int J Soc Res Methodol. 2005;8(1):1932.

8. Daudt HM, van Mossel C, Scott SJ. Enhancing the scoping study methodology: a large, inter-professional team's experience with Arksey and O'Malley's framework. BMC Med Res Methodol. 2013;13:48.

9. Levac D, Colquhoun H, O'Brien KK. Scoping studies: advancing the methodology. Implement Sci. 2010;5:69.

10. Bertani A, Menguy P, Delmas JM, et al. Assessment of surgical teleconsultations in a developing country: two years of experience in Djibouti [in French]. Med Sante Trop. 2012;22(4):405-408.

11. United Nations Development Programme. Human Development Report 2009. Accessed March 13, 2020. http://hdr.undp. org/en/reports/global/hdr2009/

12. Beer-Furlan A, Neto SG, Teixeira MJ, Figueiredo EG. Fulfilling need for neurosurgical services in sub-Saharan Africa: initial Angola-Brazil training experience. World Neurosurg. 2019;122:29-32.

13. Ploss B, Abdelgadir J, Smith ER, et al. Pilot use of a novel tool to assess neurosurgical capacity in Uganda. World Neurosurg. 2017;108:844-849.e4.

14. Guest GD, McLeod E, Perry WRG, et al. Collecting data for global surgical indicators: a collaborative approach in the Pacific region. BMJ Glob Health. 2017;2(4):e000376.

15. Haider A, Scott JW, Gause CD, et al. Development of a unifying target and consensus indicators for global surgical systems strengthening: proposed by the Global Alliance for Surgery, Obstetric, Trauma, and Anaesthesia Care (The G4 Alliance). World J Surg. 2017;41(10):2426-2434.

\section{Disclosures}

The authors report no conflict of interest concerning the materials or methods used in this study or the findings specified in this paper.

\section{Author Contributions}

Conception and design: Fuller, Barkley, Du, Elahi, Tafreshi. Acquisition of data: Fuller, Barkley, Du, Elahi, Tafreshi, Von Isenburg. Analysis and interpretation of data: Fuller, Barkley, Du, Elahi, Tafreshi, Von Isenburg. Drafting the article: Fuller, Barkley, Du, Elahi, Tafreshi. Critically revising the article: all authors. Reviewed submitted version of manuscript: all authors. Approved the final version of the manuscript on behalf of all authors: Fuller. Statistical analysis: Fuller, Barkley, Du, Elahi, Tafreshi. Administrative/technical/material support: Fuller, Von Isenburg, Haglund. Study supervision: Fuller, Haglund.

\section{Correspondence}

Anthony T. Fuller: Duke University, Durham, NC. anthony. fuller@duke.edu. 